\title{
A New Modeling Method for Objects with Branching Problem Using Non-uniform B-Spline*
}

\author{
Hyo Shin Kim ${ }^{1}$, Yoon Hyuk Kim², Yeon Hyeon Choe ${ }^{3}$, Sung-Min Kim ${ }^{4}$, \\ Taeg-Sang $\mathrm{Cho}^{5}$, and Joung Hwan Mun ${ }^{1}$ \\ ${ }^{1}$ Dept. of Bio-Mechatronic Engineering, College of Life Science \& Technology, \\ Sungkyunkwan University, Korea \\ drrose@skku.edu, jmunaskku.ac.kr \\ ${ }^{2}$ School of Advanced Technology, Kyunghee University, Korea \\ yoonhkimakhu.ac.kr \\ ${ }^{3}$ Sungkyunkwan University School of Medicine Korea \\ yhchoelsmc.samsumg. co.kr \\ ${ }^{4}$ Dept. of Biomedical Engineering, School of Medicine, Konkuk University, Korea \\ smkimakku.ac.kr \\ ${ }^{5}$ Dept. of Electrical Engineering and Computer Science, KAIST, Korea \\ taegsang@kaist.ac.kr
}

\begin{abstract}
In many applications, objects are reconstructed from cross-sections for visualization, finite element and dynamic analysis. Although cross-section of an object may contain multiple contours, a few papers have dealt with branching problem. Moreover ends of branches are described flatly. In this paper, as a basic study for dynamic analysis of a human knee joint, we present a new modeling method which proposes a data-set for solving branching problem and handling convex-end-condition of branches. We select an initial standard point from lowest slice and decide a nearest standard point of the next slice and the next, in turns. Based on standard points, we complete the data-set by applying contour alignment. For 3D reconstruction, the surface is approximated by bicubic non-uniform B-spline surface fitting. This method provides the smooth surface model with $\mathrm{C}^{2}$ continuity and describes the convexity of ends of branches.
\end{abstract}

\section{Introduction}

In many applications, objects are often reconstructed from 2D cross-sections for visualization of 3D structure, finite element and multibody dynamic analysis. For 3D reconstruction, several methods have been proposed, ranging from polyhedron-based approaches[1, 3, 4, 5], intensively studied during the past decade, to tiling algorithms $[1,3,4,5]$, skinning methods $[6,7,8]$ and surface fittings $[2,9,10,13]$.

\footnotetext{
* This study was supported by a grant of the Korea Health 21 R\&D Project, Ministry of Health \& Welfare, Republic of Korea.(02-PJ3-PG6-EV06-0002)

Corresponding author: Joung H. Mun, Ph.D., Dept. of Bio-Mechatronic Engineering, College of Life Science and Technology, Sungkyunkwan University, Suwon, Korea. (Tel) +82-31290-7827 (e-mail)jmun@skku.ac.kr
} 
In general, each cross-section may contain multiple contours. Some studies have nevertheless proposed branching algorithms to link one or more contours in one cross-section to multiple contours in an adjacent cross-section with triangular facets $[1,4,5]$. And only a few papers have approximated a set of $2 \mathrm{D}$ contours with branching problems. Park and Kim[10] proposed a method for B-spline surface approximation to a set of contour with branching problem but triangular facets were constructed over each branching region and built triangular surface patches over these facets. Jeong et al[2] proposed B-spline surface approximation to cross-sections using distance maps. This method provided a smooth surface model, yet realized efficient data reduction and described ends of branches as a flat surface by using the end tangent condition[2, 11]. However this flat surface affects seriously force recovery if this model is applied to dynamic analysis of the joint, because contact points occurring at the joint are usually placed on the flat surface.

In this paper, we present a new modeling method for objects with branching problems which uses a data-set for solving branching problem and handling convexend-condition of branches simultaneously. For 3D reconstruction, the surface is approximated by tensor product non-uniform B-spline surface fitting. This method provides bicubic non-uniform B-spline surface with $\mathrm{C}^{2}$ continuity and describes the convexity of ends of branches.

\section{Surface Fitting}

If an object has complex shape, double branching situation occurs frequently[5]. So in this paper, we consider objects with double branching. Double branching is a case in which a contour $C_{r}^{k+1}$ at level $z_{k+1}$ must be linked to two contours, $C_{i}^{k}$ and $C_{j}^{k}$, at its lower level $z_{k}$ shown in the Fig. 1. Here, data points have a set of heights $z_{0}<z_{1}<\ldots<$ $z_{k}<\ldots<z_{\max }$ and points of the $k$ th contour are saved counterclockwisely.

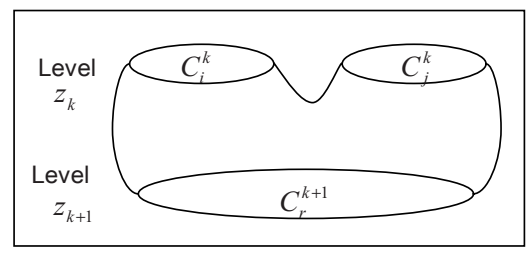

Fig. 1. Definition of double branching problem

The proposed method consists of three main phases, data-set, contour alignment, surface fitting. First, we briefly review definitions of B-spline curve and surface fitting. A detailed discussion of B-spline theory can be found in the literature[11].

\subsection{Non-uniform B-Spline Curve Fitting}

As a foundation for the surface fitting algorithm, we first apply open non-uniform Bspline curve fitting to contours. Here, a cubic B-spline is employed as the basis 
function $(p=3)$ and satisfies $Q_{0}=\mathrm{C}(0)$ and $Q_{m}=\mathrm{C}(1)$. In order to fit the open B-spline curve to given $m+1$ contour points $Q_{k}$, we use the least squares formulation below to find control points $P_{i}$ which is unknown as $n+1$ and assume $n \geq p$.

$$
f=\sum_{k=1}^{m-1}\left|Q_{k}-C\left(\bar{u}_{k}\right)\right|^{2}
$$

is a minimum with respect to $n+1$ variables, $P_{i} . f$ is a scalar-valued function of $n-1$ variables, $P_{1}, \ldots, P_{n-1}$. To minimize $f$, we set derivatives of $f$ with respect to $n$-1 points, $P_{l}$, equal to zero. Finally one linear equation remains below and we can calculate control points $P_{1}, \ldots, P_{n-1}$.

$$
\left(N^{T} N\right) P=R
$$

The $\bar{u}_{k}$ and $U$ affect the shape and parameterization. The $\bar{u}_{k}$ is the parameter value precomputed by the chord length method[11] which is most widely used and generally adequate.

The placement of knots should reflect the distribution of the $\bar{u}_{k}$. We need a total of $n+p+2$ knots and there are $n-p$ internal knots, and $n-p+1$ internal knot spans. Let

$$
t=\frac{m+1}{n-p+1}
$$

then define internal knots by

$$
\begin{gathered}
i=\operatorname{int}(j t), \quad \alpha=j t-i, \\
u_{p+j}=(1-\alpha) \bar{u}_{i-1}+\alpha \bar{u}_{i}, \quad j=1, \ldots, n-p .
\end{gathered}
$$

This guarantees that every knot span contains at least one $\bar{u}_{k}$, and under this condition the matrix $\left(N^{T} N\right)$ is positive definite and well-conditioned. It can by solved by Gaussian elimination without pivoting.

\subsection{Tensor Product Non-uniform B-Spline Surface Fitting}

The final step is to generate a bicubic open non-uniform B-spline surface and the resulting B-spline surface is expressed as

$$
S(u, v)=\sum_{i=0}^{n} \sum_{j=0}^{m} N_{i, p}(u) N_{j, q}(v) P_{i, j}
$$

where $P$ and $S$ have $x, y$ and $z$ components. The control points $P_{i, j}$ are $(n+1)$ by $(m+1)$ network in $u$ and $v$ directions and we also calculate these by least squares method defined by

$$
\sum_{k=1}^{r-1} \sum_{l=1}^{s-1}\left|Q_{k, l}-S\left(\bar{u}_{k}, \bar{v}_{l}\right)\right|^{2} .
$$

Again, the first order of business is to compute reasonable values for the $\left(\bar{u}_{k}, \bar{v}_{l}\right)$ and knot vectors $U$ and $\mathrm{V}$. A common method like the curve fitting is used to compute 
parameters $\bar{u}_{0}^{l}, \ldots, \bar{u}_{r}^{l}$ for each $l$, and then each $\bar{u}_{k}$ is obtained by averaging across all $\bar{u}_{k}^{l}, l=0, \ldots, \mathrm{s}$, and $\bar{v}_{l}$ is also calculated. Once $\left(\bar{u}_{k}, \bar{v}_{l}\right)$ are computed, knot vectors $U$ and $V$ can be obtained like the method at Sect. 2.1 .

\subsection{Branching Problem and Convex-End-Condition Handling; Data-Set}

It is simple to construct a data-set for reconstructing a geometric model of an object with double branching problem. The number of points of left and right branch extracted from branches must be same and the number of row and column of points at one side (left side in this paper) of branches are followed;

$$
\begin{aligned}
& \text { Number of row at one side of branch }=x+2 \times(n-1), \\
& \text { Number of column at one side of branch }=2 \times(2 \times n)
\end{aligned}
$$

where $x$ and $n(=1,2, \ldots)$ mean a quarter of the total number of points of slice at the $z_{0}$ and the slice number, respectively. And the total number of points demanded at the first base and the number of row and column of points at the base are followed;

Total number of points at the first base

$$
\begin{gathered}
=2 \times\left\{x+2 \times\left(n_{\max }-1\right)+2 \times m_{1}\right\}+2 \times\left(4 \times n_{\max }+2 \times m_{1}\right)-4, \\
\text { Number of row at the base }=x+2 \times\left(n_{\max }-1\right)+2 \times m, \\
\text { Number of column at the base }=4 \times n_{\max }+2 \times m
\end{gathered}
$$

where $n_{\max }$ is the number of $\operatorname{slice}(=\mathrm{k}+1)$ at the $z_{k}, m_{l}(=1)$ and $m(=1,2, \ldots)$ mean the slice at the $z_{k+1}$ and the number of slice counted only at the base, respectively. Thus the size of data-set is $\left(x+2 \times\left(n_{\max }-1\right)+2 \times m, 4 \times n_{\max }+2 \times m\right)$. Here each value of column includes three components, $\mathrm{x}, \mathrm{y}$ and $\mathrm{z}$.

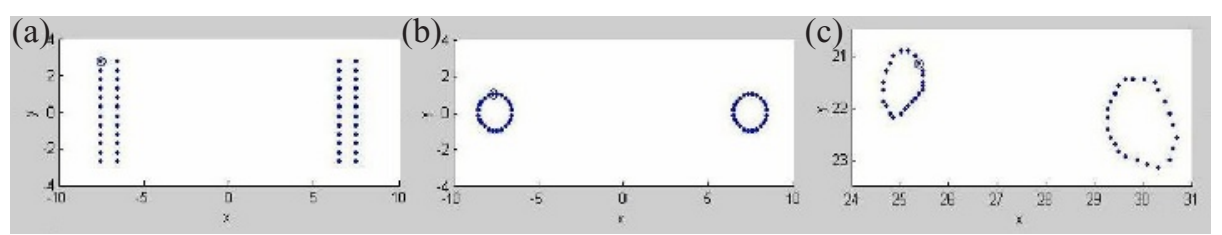

Fig. 2. Initial standard point (surrounded by small circle), (a) rectangle-based object( $R B O)$, (b) circle-based object $(C B O)$ and (c) femur

For constructing the data-set, we select an initial standard point from a slice at the $z_{0}$. As shown in Fig. 2, it is efficient to select a maximum(or minimum) point placed at the perpendicular direction to the line passing through approximate centers of two branches at the left branch or a nearby point of a mentioned point. Based on this point, the others are rearranged counterclockwisely. Points from branches and bases are located at the left and right side inside of a data-set and outside of a data-set to surround points from branches, respectively 


\subsection{Contour Alignment}

As the control net of a B-spline surface is composed of a set of control polygons, a number of different control nets can be defined by variously aligning the given control polygons. A different alignment of control polygons gives rise to a different control net and results in the different shape of the surface. Since points of $u$ - and $v$ direction pass through different slices, neighboring points have to be arrayed as closely as possible. After an initial standard point is selected by Sect. 2.3 we select a shortest one of the next slice. Then this point becomes a standard point of the next slice. Like this way, standard points are selected from all of slices. Standard points are located at the closest place from a previous standard point, just above. By the contour alignment, we can prevent unwanted twists or wiggles.

\subsection{Approximation to within a Specified Accuracy}

The bicubic B-spline surface can be obtained by (5) and the sum-of-squares error can be calculated by comparing surface points at assigned data parameter values to original data points[12], as shown by

$$
E(P)=\sum_{k=0 l=0}^{r} \sum_{l=0}^{s}\left(\left(\sum_{i=0}^{n} \sum_{j=0}^{m} N_{i, p}\left(\bar{u}_{k}\right) N_{j, q}\left(\bar{v}_{l}\right) P_{i, j}\right)-Q_{k, l}\right)^{2} .
$$

The average error in the surface fit is given by

$$
E_{\text {ave }}(P)=\frac{1}{(r+1)(s+1)} E(P) \text {. }
$$

To obtain the approximating data to within some specified error bound, E, we use iterative method which have three steps:

1. Starting with $(p+1, q+1)$ control point (the minimum) and fit a surface.

2. Checking if the surface deviation is less than E. A record is maintained for each knot span, indicating whether or not it has converged.

3. If the surface deviation does not satisfy $E$, a knot is added at the mid point of each nonconvergent span, thus adding control points. And if the deviation is less than $E$, stopping the reiteration.

\section{Experimental Results}

The proposed data-set has been applied to three different sets of contour data. We use the synthetic data of rectangle-based object(RBO) and circle-based object(CBO) to verify the method and the CT data of inferior region of a femur as a general case with double branching problem.

The information of two synthetic data and CT data is denoted in Table 1 and their cross-sections are shown in Fig. 3. In Table 1, the size of data-set, (row $\times$ column), 
means total number of points which have $\mathrm{x}, \mathrm{y}$, and $\mathrm{z}$ components. In Fig. 3 , the contour in each cross-section has been segmented and compressed into a closed polygon. And small circles on a red line passing through contours are standard points of each contour by contour alignment, starting at an initial standard point of lowest slice.

Table 1. Information of two synthetic data and CT data of a femur

\begin{tabular}{|cccc|}
\hline & RBO & CBO & Femur \\
\hline $\begin{array}{c}\text { Number of } \\
\text { slices } \\
\text { Distance } \\
\text { between } \\
\text { neighboring } \\
\text { slices } \\
\text { Size of data-set }\end{array}$ & 14 & 14 & 18 \\
\hline
\end{tabular}

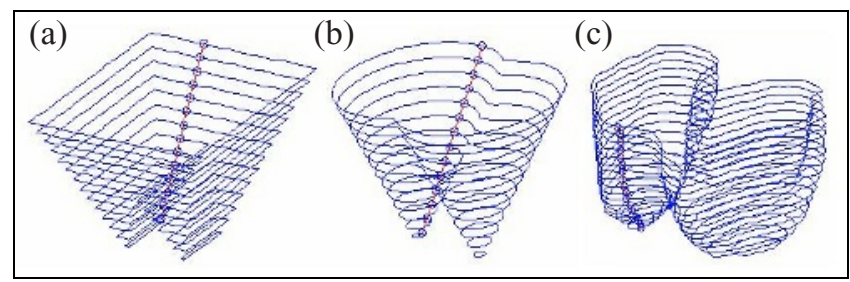

Fig. 3. Cross-sections of (a) RBO, (b) CBO and (c) femur

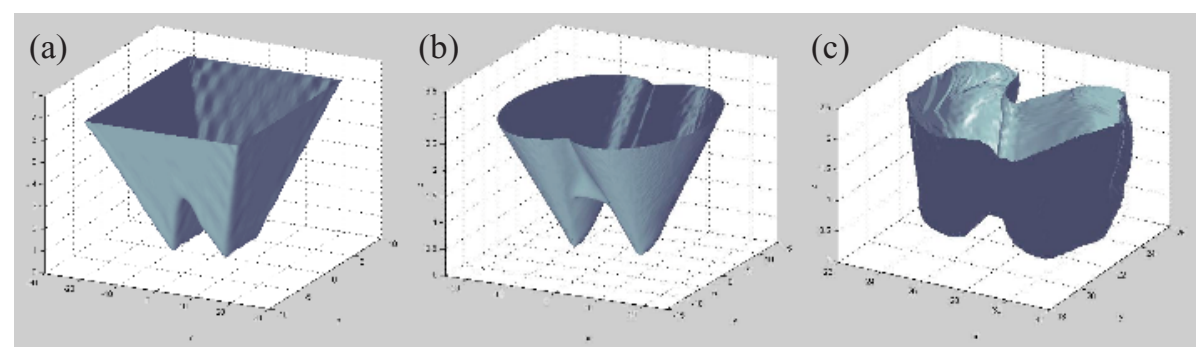

Fig. 4. B-spline surface of (a) RBO, (b) CBO and (c) femur

Table 2. Number of control points, total error and average error

\begin{tabular}{cccc|}
\hline & RBO & CBO & Femur \\
\hline $\begin{array}{c}\text { Number of control points } \\
(u \text { direction, } v \text { direction })\end{array}$ & $(20,21)$ & $(19,21)$ & $(16,19)$ \\
$\mathrm{E}(\mathrm{P})$ & 827.0186 & 796.4465 & 88.2719 \\
$\mathrm{E}_{\mathrm{ave}}(\mathrm{P})$ & 1.9691 & 1.9961 & 0.2904 \\
\hline
\end{tabular}

Figure 4 shows shaded surfaces. The resulting surface is approximated by bicubic non-uniform B-spline surface. Table 2 presents the number of control points fitted in 
given accuracy, a total error and an average error occurring at data points with respect to the surface. In the number of control points, ( $u$ direction, $v$ direction), $u$ direction and $v$ direction mean the number of points needed to approximate the vertical and horizontal data of a data-set, respectively.

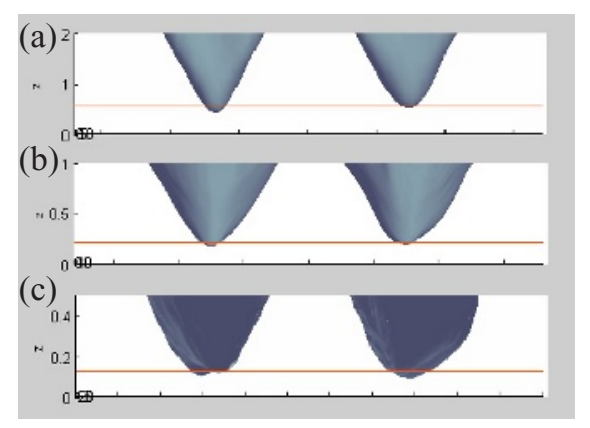

Fig. 5. Ends of branches, (a) RBO, (b) $\mathrm{CBO}$ and (c) femur

Figure 5 shows states of ends of branches and each example has double branches. A straight red line means a lowest slice, $\mathrm{n}_{1}$, and $\mathrm{z}$ values are $0.5,0.2$ and 0.125 for (a) $\mathrm{RBO}$, (b) $\mathrm{CBO}$ and (c) femur, respectively. If there is just a previous slice of a lowest slice, its $\mathrm{z}$ value is 0 for all examples. As shown in figure, ends of branches are reconstructed convexly and its surface is smooth for all direction. We can also verify lowest points for all ends of branches are within $\mathrm{z}$ value, 0 .

\section{Conclusions and Discussions}

As a basic study for more advanced dynamic analysis of knee joint, we have presented a new modeling method for objects with branching problem. For constructing a data-set, we select an initial standard point and complete the data-set based on standard points by applying contour alignment. For 3D reconstruction, the surface is approximated by tensor product non-uniform B-spline surface fitting. This method has provided a smooth bicubic B-spline surface model with $\mathrm{C}^{2}$ continuity, as shown in Fig. 4.

In addition to a surface approximation, another important fact for surface fitting is the error between data points and points on the surface. Control points are calculated by least squares sense so that the surface is closer to the data. The more the number of control points increases, the more approximation improves. However, as the number of control points approaches the number of data points, noises or unwanted wiggles can occur according to [11]. So the tolerance for an average error allowable was 2.0 for $\mathrm{RBO}$ and $\mathrm{CBO}$ and 0.3 for a femur which are determined empirically. As shown in Table 2 and Fig. 4, average errors are satisfied with the given tolerance and resulting surface models have no noises or unwanted wiggles.

Moreover, as shown in Fig. 5, ends of branches of all examples are convex and within the distance between neighboring slices. Sun et al[13] used 'doom' feature in SolidWorks to describe the terminal region convexly for finite element analysis or multibody dynamic analysis of human middle ear. Thus convex-end-condition is very 
important and we think these results will play an important role in conducting more advanced dynamic analysis of knee joint hereafter.

The number of points extracted from images is restricted within small number if the resolution of images is low. This can be limitation but we can overcome this problem by improvements in data acquisition and imaging techniques such as computed tomography, magnetic resonance imaging and ultrasound imaging. Since the location of points affects significantly to the shape of a model, the data-set must not have sharp turn. When there are sharp turns, it need to use the centripetal method for parameter values or rise the degree of B-spline, as described in the literature[11].

We concluded this new method provided $\mathrm{C}^{2}$ continuous bicubic B-spline surface model of objects including branching problem and it described the convex surface, required absolutely for dynamic analysis of joints, occurring at ends of branches smoothly within distance between neighboring slices. In the future study, we have a plan to execute more advanced dynamic analysis of a human knee joint.

\section{References}

1. Park, H., Kim, K.: 3D shape reconstruction from 2D cross sections. Journal of Design and Manufacturing. 5 (1995) 171-185

2. Jeong, J., Kim, K., Park, H., Cho, H., Jung, M.: B-Spline Surface Approximation to CrossSections Using Distance Maps. Advanced Manufacturing Technology. 15 (1999) 876-885

3. Zyda, M., Allan, R., Hogan, P.: Surface construction from planar contours. Computers and Graphics. 11(4) (1987) 393-408

4. Ekoule, A., Peyrin, F., Odet, C.: A triangulation algorithm from arbitrary based multiple planar contours. ACM Transactions on Graphics. 10(2) (1992) 182-199

5. Choi, Y., Park, K.: A heuristic triangulation algorithm for multiple planar contours using an extended double branching procedure. Visual Computer. 10 (1994) 372-387

6. Woodward, C.: Skinning techniques for interactive B-spline surface interpolation. Computer Aided Design. 20(8) (1988) 441-451

7. Odesanya, O., Waggenspack, W., Thompson, D.: Construction of biological surface models from cross-sections. IEEE Transactions of Biomedical Engineering. 40(4) (1993) 329-334

8. Kaklis, P., Ginnis, A.: Sectional-curvature preserving skinning surfaces. Computer Aided Geometric Design. 13(7) (1996) 601-619

9. Rogers, D., Fog, N.: Constrained B-spline curve and surface fitting. Computer-Aided Design. 21(10) (1989) 641-648

10. Park, H., Kim, K.: Smooth surface approximation to serial cross-sections. ComputerAided Design. 28(12) (1996) 995-1005

11. Piegel, L. and Tiller, W.: The NURBS Book. Springer Berlin (1995)

12. Klingensmith, J., Vince, D.: B-spline methods for interactive segmentation and modeling of lumen and vessel surfaces in three-dimensional intravascular ultrasound. Computerized Medical Imaging and Graphics. 26 (2002) 429-438

13. Sun, Q., Chang, K., Dormer, K., Dyer, R. Jr., Gan, R.: An advanced computer-aided geometric modeling and fabrication method for human middle ear. Medical Engineering and Physics. 24 (2002) 595-606 